\title{
miR-34c disrupts spermatogonial stem cell homeostasis in cryptorchid testes by targeting Nanos2
}

Zhenyu Huang ${ }^{1 \dagger}$, Dongdong Tang ${ }^{2,3,4 \dagger}$, Jingjing Gao ${ }^{1}$, Xianming Dou' ${ }^{1}$, Peng Cheng ${ }^{1}$, Dangwei Peng ${ }^{1}$, Yao Zhang ${ }^{1}$, Jun Mao', Li Zhang ${ }^{1}$ and Xiansheng Zhang ${ }^{1 *}$

\begin{abstract}
Background: Cryptorchidism as a common genitourinary malformation with the serious complication of male infertility draws widespread attention. With several reported miRNAs playing critical roles in spermatogonial stem cells (SSCs), we aimed to explore the fundamental function of the highly conserved miR-34c in cryptorchidism.

Methods: To explore whether miR-34c participates in spermatogenesis by regulating Nanos2, we examined the effect of overexpression and inhibition for miR-34c on Nanos2 expression in GC-1 cells. Moreover, the expression levels of miR-34c and Nanos2 with cryptorchidism in humans and mice were examined. Furthermore, the homeostasis of SSCs was evaluated through counting the number of promyelocytic leukemia zinc finger (PLZF) positive spermatogonia in murine cryptorchid testes.

Results: In the present study, we show that miR-34c could inhibit the expression of Nanos2 in GC-1 cells. Meanwhile, miR-34c significantly decreased in both the testicular tissues of patients with cryptorchidism and surgery-induced murine model of cryptorchidism. Western blot revealed that the protein level of Nanos 2 was up-regulated and showed to be negatively correlated to the expression of miR-34c in our model. The abnormal expression of miR-34c/Nanos2 disrupted the balance between SSC self-renewal and differentiation, eventually damaging the spermatogenesis of cryptorchid testes.

Conclusions: The miR-34c/Nanos2 pathway provides new insight into the mechanism of male infertility caused by cryptorchidism. Our results indicate that miR-34c may serve as a biological marker for treatment of infertility caused by cryptorchidism.
\end{abstract}

Keywords: Cryptorchidism, miR-34c, Nanos2, Spermatogonial stem cell

\section{Background}

Male infertility is a serious health problem that has been receiving more and more attention around the world $[1,2]$. Idiopathic diseases of the male reproductive system, such as cryptorchidism and varicocele, are important factors that lead to male infertility $[3,4]$. As a common genitourinary malformation, cryptorchidism is a condition that occurs due the continual exposure of the undescended testis to elevated temperatures

\footnotetext{
* Correspondence: xiansheng-zhang@163.com

†'Zhenyu Huang and Dongdong Tang contributed equally to this work.

'Department of Urology, The First Affiliated Hospital of Anhui Medical

University, Hefei 230022, China

Full list of author information is available at the end of the article
}

because of its location in the abdominal cavity or canalis inguinalis, culminating in serious damage to spermatogenesis. Spermatogonial stem cells (SSCs), which possess self-renewal capacity and could differentiate into spermatozoa, are the foundation of spermatogenesis [5]. The balance between self-renewal and differentiation in SSCs ensures stable and persistent spermatogenesis in the testes. Nanos2, an evolutionarily conserved RNA binding protein, is the marker of undifferentiated spermatogonia and is found to be almost exclusively expressed in $A_{s}$ to $\mathrm{A}_{\mathrm{pr}}$ subpopulation of spermatogonia [6]. Recent studies have reported that Nanos2-mediated post-transcriptional regulation is essential for homeostasis of murine SSCs. Nanos2 maintains SSC homeostasis by the translational

(c) The Author(s). 2018 Open Access This article is distributed under the terms of the Creative Commons Attribution 4.0 International License (http://creativecommons.org/licenses/by/4.0/), which permits unrestricted use, distribution, and 
repression of differentiation-related mRNAs and the sequestration of the mTOR protein [7]. In contrast, highly expressed Nanos 2 resulted in the accumulation of promyelocytic leukemia zinc finger (PLZF) positive spermatogonia and the destruction of SSC balance [8].

MicroRNAs (miRNAs), small non-coding RNA molecules of $\sim 22$ nucleotides, target the 3 '-untranslated region (3'UTR) of mRNAs to regulate protein expression in various biological processes [9]. A number of miRNAs, such as miR-17, miR-18, miR-19 and miR-25, have been demonstrated to play critical roles in spermatogenesis. They contribute to early spermatogonial differentiation and deficiency of the miR-34 family leads to mouse infertility [10]. miR-34c, belonging to the highly conserved miR-34 family across diverse species, was reported to be associated with proliferation, apoptosis and invasion of multiple malignancies, such as breast cancer and endometrial carcinoma [11-13]. Moreover, miR-34c is preferentially expressed in the testes of mice and participates in spermatogenesis by regulating targets. For example, miR-34c enhances male germ cell apoptosis through targeting ATF1 and regulates murine testicular development and spermatogenesis by targeting the E2F-pRb pathway $[14,15]$. Furthermore, simultaneous inactivation of miR-34b/c and miR-449 loci resulted in oligoasthenoteratozoospermia and infertility in male mice $[16,17]$. A previous study showed that miR-34c was abundantly expressed in SSC-enriched germ cell cultures and could enhance SSC differentiation [18]. Although the function of miR-34c was largely explored in multiple malignancies and SSCs, whether miR-34c is involved in congenital genitourinary malformations, particularly cryptorchidism, remains unclear.

To address this question, we checked the expression level of miR-34c in cryptorchidism and explore its underlying mechanisms. We found that miR-34c was significantly decreased in the testicular tissues of patients with cryptorchidism. Moreover, the downregulation of miR-34c increases the protein level of Nanos2 to disrupt SSC homeostasis in murine cryptorchid testes. The miR-34c/ Nanos 2 pathway may provide new insight into the mechanism of male infertility caused by cryptorchidism.

\section{Methods}

\section{Human tissue samples}

Human testicular tissues were obtained from the First Affiliated Hospital of Anhui Medical University (Hefei, China). All patients, whose ages ranged from 18 to 33 years old, signed an informed consent, and their tissues were allowed for research purposes. Six samples of testicular tissue from patients with cryptorchidism and another six samples of testicular tissue from patients with male infertility caused by obstructive azoospermia (OA) were collected for comparison. This study was approved by the
Ethics Committee of the First Affiliated Hospital of Anhui Medical University.

\section{Cell culture, plasmid construction and transfection}

GC-1 cell lines were obtained from BeNa Culture Collection (Bncc Biotech, Beijing, China) and maintained according to their recommendations. The plasmid for overexpressing mouse miR-34c was constructed with recombinant methods (Vazyme c113-02). Firstly, the vector named pmR-mCherry (ClonTech) was linearized with EcoRI and BamHI. Then the amplified fragment was purified and inserted into the backbone. Primer sequences were as followed: forward: 5'-CCGGAAACTAGTCTCA GATCTGCTGTGTGGTTAGTGATTGG-3' and reverse: 5'-GATTATGATCAGTTATCTAGATACCTGTGTGTAA GAGCCAG-3'. The miR-34c inhibitor was synthesized by Ribobio (Ribobio Biotech, Guangzhou, China). Plasmid transfection was conducted with Lipofectamine 2000 (Invitrogen) according to the manufacturer's protocol with G418 selection $(400 \mu \mathrm{g} / \mathrm{mL})$. Transfection of RNA inhibitor was conducted with Oligofectamine (Invitrogen) according to the manufacturer's protocol.

\section{Animals}

All mice were handled in accordance with the relevant guidelines, and all experimental procedures were approved by the Ethics Committee of Anhui Medical University. A total of 60 male ICR mice, 6-weeks-old, were obtained from the Laboratory Animal Centre of Anhui Medical University and kept in a specific pathogen-free animal facility. The mice were exposed to a $14 \mathrm{~h}$ light/ $10 \mathrm{~h}$ dark cycle and maintained at a suitable temperature of $22 \pm 2{ }^{\circ} \mathrm{C}$ with free access to food and water.

\section{Mouse model of cryptorchidism}

Male ICR mice were randomly divided into two groups: the cryptorchidism group $(n=30)$ and the control group $(n=30)$. The skin of the left lower abdomen was disinfected after the mice were anaesthetized. In the cryptorchidism group, the left gubernaculum was sheared after opening the abdominal cavity, followed by suturing of the fat pad near the testes with peritoneum to fix the left testes in the abdominal cavity. In the control group, after opening the abdominal cavity, the left testes were gently pulled and left in the original scrotal position. No treatments were performed on the right testes in both groups. Mice were sacrificed by cervical dislocation at 3, 7, 14, 21 and 28 days after the operation was completed. The testes were obtained, then photographed and weighed.

\section{Histological analysis and immunohistochemistry}

Testicular tissues were fixed with $4 \%$ paraformaldehyde, then embedded in paraffin and divide into $4-\mu \mathrm{m}$ sections. To evaluate the histological changes of cryptorchid 
testes, the sections were stained with hematoxylin and eosin (HE)following a standard protocol. Furthermore, the location and expression of PLZF were determined by immunohistochemistry to assess the changes in the number of spermatogonia. The testicular tissue sections were heated in sodium citrate buffer $(\mathrm{pH}$ 6.0) for 2 mins and then dipped in deionized water containing 3\% $\mathrm{H} 2 \mathrm{O} 2$ for 20 mins to quench endogenous peroxidase activity. Next, the sections were incubated with PLZF-specific antibody (1:20; Santa Cruz Biotech, Dallas, TX, USA) at $4{ }^{\circ} \mathrm{C}$ overnight and then incubated with horseradish peroxidase-labelled secondary antibody at $37{ }^{\circ} \mathrm{C}$ for 30 mins. Finally, the sections were stained with diaminobenzidine and counterstained with Harris's hematoxylin.

\section{Real-time RT-PCR}

Total RNA was extracted from testicular tissues with TRIzol reagent (Invitrogen, Thermo Fisher Scientific, Inc., Foster City, CA, USA) following the manufacturer's protocol. Complementary DNA was synthesized from RNA with the FastQuant RT Kit (Tiangen Biotech, Beijing, China) according to the manufacturer's protocol. The primer of miR-34c used for cDNA synthesis was GTCG TATCCAGTGCAGGGTCCGAGGTATTCGCACTGGA TACGACGCAATC. Real-time PCRs were performed with the SuperReal PreMix Plus (SYBR Green) Kit in an ABI7500 Real Time PCR System (Applied Biosystems; Thermo Fisher Scientific, Inc.). In addition, melting-curve analysis was used to monitor the purity of the PCR product. The expression levels of mature miR-34c and Nanos2 mRNA were normalized to $18 \mathrm{~S}$ rRNA. The primer sequences are listed in Table 1.

\section{Western blotting}

For preparation of samples, protein extracted from testicular tissues was minced and lysed in RIPA buffer (Beyotime Biotech, Shanghai, China) on ice more than 20 mins. After sonication, the concentrations were measured with a BCA protein assay kit (Beyotime Biotech). Total protein was loaded onto 15\% SDS-PAGE gels and then transferred to PVDF membranes. Membranes were processed according to the ECL Western blotting protocol (GE Healthcare, General Electric Company, Boston, USA). Quantification of the band intensities was performed with Image (NIH, Bethesda, MD, USA). An area above each band, the same size as the corresponding band, was used for background subtraction. The following antibodies were used in Western blots: anti-Nanos2 (1:200; GeneTex, Irvine, CA, USA) and anti-GAPDH (1:1000; TransGen Biotech, Beijing, China).

\section{Statistical analyses}

The values shown in the graphs represent averages of at least three independent experiments, with error bars showing standard deviations. Two-sample Student's t tests and Wilcoxon rank sum tests were carried out using SPSS version 22.0 (SPSS, Chicago, IL, USA). $P<0.05$ was considered significantly different.

\section{Results}

miR-34c decreases in the testicular tissues of patients with cryptorchidism

We investigated whether or not miR-34c functioned in the testicular tissues of patients with cryptorchidism. Six samples of testicular tissue from patients with cryptorchidism and another six samples of testicular tissue from patients with OA were used for comparison. Histological examination showed significant difference between the two groups (Fig. 1a). The Johnsen score was performed to evaluate the spermatogenesis of testes. The Johnsen scores of patients with cryptorchidism were just 2-3 scores (data not shown). Per real-time PCR, the expression level of miR-34c significantly decreased in the testicular tissues of patients with cryptorchidism compared to that of patients with OA $(1.19 \pm 0.633$ vs 0.0292 $\pm 0.0207, P=0.006$ ) (Fig. 1b).

\section{miR-34c inhibits Nanos2 expression in GC-1 cells}

To investigate the roles of miR-34c in cryptorchidism, we focused on its mechanism of function. Using online prediction tools, such as TargetScan and miRDB, we found several targets of miR-34c, among which was Nanos2, a gene with great significance in SSC homeostasis. To explore whether miR-34c participates in spermatogenesis by regulating Nanos2, we examined the effect of overexpression and inhibition for miR-34c on Nanos2 expression in GC-1 cells. After transfecting the plasmid of miR-34c for $48 \mathrm{~h}$ in GC-1 cell, RT-qPCR showed the markedly increase of miR-34c level. RT-qPCR and western blot analysis demonstrated significant decrease of Nanos2 expression level $(1.00 \pm 0.0830$ vs $3.85 \pm 0.486$,

Table 1 The primer sequences used for Real-time RT-PCR

\begin{tabular}{llll}
\hline Gene & Species & Forward & Reverse \\
\hline Nanos2 & Mouse & CCATATGCAACTTCTGCAA & ACTGCTGACTGCTGTTGAGTG \\
Nanos2 & Human & CTGAGAAGTGCCTACTCAAG & TGATACGGTGCTCTCCAGAG \\
miR-34c & Human/Mouse & CACGCAAGGCAGTGTAGT & CCAGTGCAGGGTCCGAGGTA \\
185 & Human/Mouse & CGGCGACGACCCATTCGAAC & GAATCGAACCCTGATTCCCCGTC \\
\hline
\end{tabular}




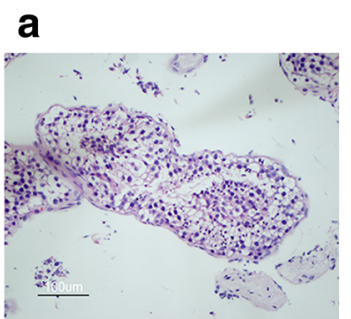

$\mathrm{OA}$
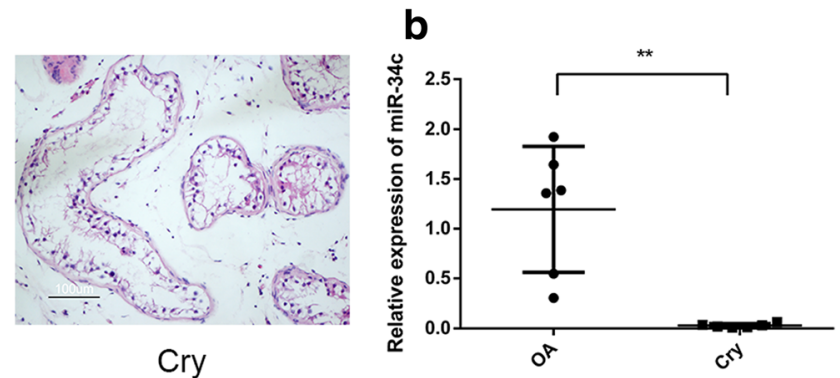

Fig. 1 miR-34c was markedly reduced in the testicular tissues of patients with cryptorchidism. a The histological comparison of testicular tissues between cryptorchidism patients and OA patients. b The expression level of miR-34c in the testicular tissues of patients with cryptorchidism. OA patients were the control. Bar $=100 \mu \mathrm{m}$. OA: obstructive azoospermia; Cry: cryptorchidism. ${ }^{* *} P<0.01$

$P=0.008 ; \quad 1.00 \pm 0.0642 \quad$ vs $0.556 \pm 0.0483, \quad P=0.001)$ (Fig. 2a, c). In reverse, when treated with the inhibitor of miR-34c, GC-1 cells showed the down-regulation of miR-34c and up-regulation of Nanos2 expression level $(1.00 \pm 0.0871$ vs $0.401 \pm 0.0208, P<0.001 ; 1.00 \pm 0.119$ vs $1.78 \pm 0.105, P=0.001$ ) (Fig. 2b, c). All the results indicate that miR-34c could inhibit the expression of Nanos2 in GC-1 cells.

\section{Establishment of mouse model of cryptorchidism}

To further explore the role of miR-34c in cryptorchidism, we established a surgery-induced mouse model of cryptorchidism, as previously described [19, 20]. In the cryptorchidism group, the left testes, known as the treated side testes, of mice were fixed in the abdominal cavity to simulate an environment of cryptorchidism and the contralateral ones as the untreated side testes, serving as self-control. We also performed the operation on the left lower abdomen to exclude the influence of surgical trauma as group control. The contrast of testes between the cryptorchidism group and control group for 3, 7, 14, 21 and 28 days after operation are shown in Fig. 2a. Furthermore, the weight percentage of testes compared to the whole body was calculated as the testis index to evaluate the difference of testes of the two groups. We found that the treated side testes of the cryptorchidism group significantly decreased both in volume and in weight compared with the untreated side testes and control group at 7, 14, 21 and 28 days after operation ( 3 days: Con vs $T=0.390 \pm 0.0617$ vs $0.384 \pm$ $0.0829, P=0.882$; UT vs $T=0.394 \pm 0.0529$ vs $0.384 \pm$ $0.0829, P=0.795$. 7 days: Con vs $T=0.359 \pm 0.0461$ vs $0.258 \pm 0.0761, P=0.019 ;$ UT vs $T=0.386 \pm 0.0385$ vs $0.258 \pm 0.0761, P=0.007$. 14 days: Con vs $T=0.330 \pm$ 0.0364 vs $0.165 \pm 0.0629, P<0.001$; UT vs $T=0.383 \pm$ 0.0591 vs $0.165 \pm 0.0629, P<0.001 .21$ days: $C o n$ vs $T=$ $0.322 \pm 0.0237$ vs $0.116 \pm 0.0389, P<0.001$; UT vs $T=$ $0.350 \pm 0.0792$ vs $0.116 \pm 0.0389, P<0.001$. 28 days: Con vs $T=0.340 \pm 0.527$ vs $0.140 \pm 0.0539, P<0.001$; UT vs $T$ $=0.333 \pm 0.0632$ vs $0.140 \pm 0.0539, P<0.001)$ (Fig. 3a, b). a

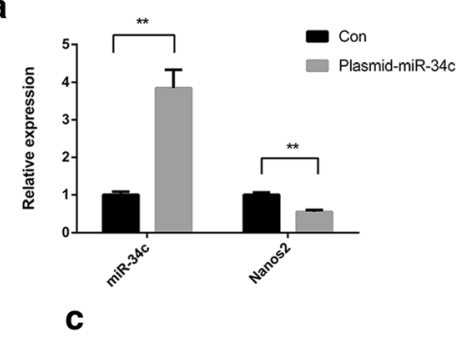

Con Plasmid-miR-34c

Nanos2

GAPDH b

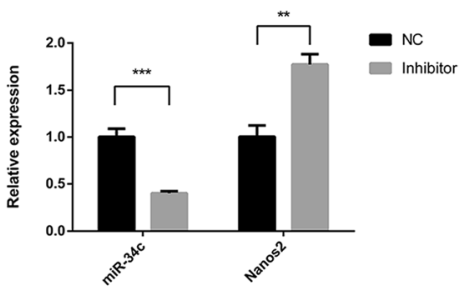

NC Inhibitor

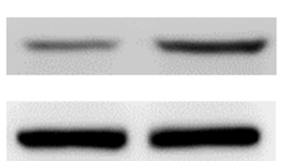

Fig. 2 miR-34c inhibit the expression of Nanos2 in GC-1 cells. a Relative expression levels of miR-34c and Nanos2 mRNA were analyzed by RT-qPCR after transfecting the plasmid of miR-34c in GC-1 cell. b Relative expression levels of miR-34c and Nanos2 mRNA were analyzed by RT-qPCR after miR-34c inhibition in GC-1 cell. c Nanos2 protein levels in a GC-1 cell culture after miR-34c overexpression or inhibition. Con: control; NC: negative control. ${ }^{*} P<0.05,{ }^{* *} P<0.01$ and ${ }^{* * *} P<0.001$ 
a
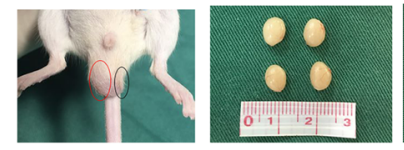

3 days

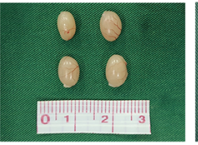

7 days

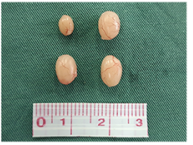

14 days

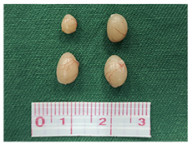

21 days

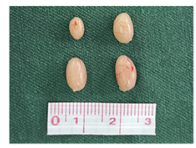

28 days

b
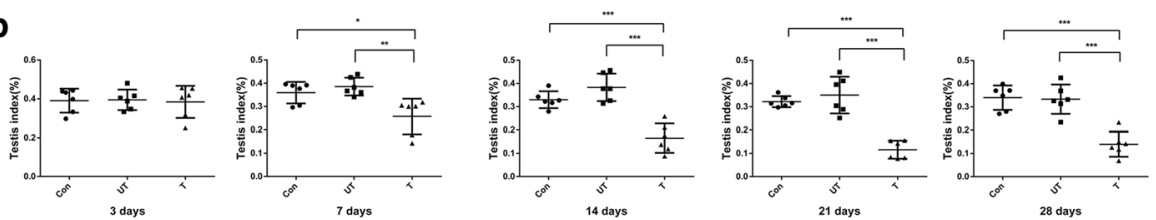

C

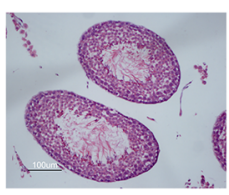

Con

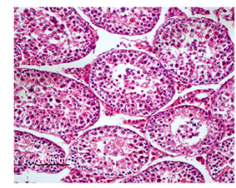

14 days

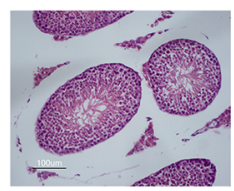

UT

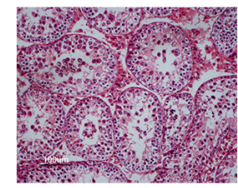

21 days

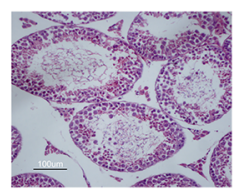

3 days

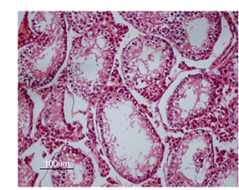

28 days

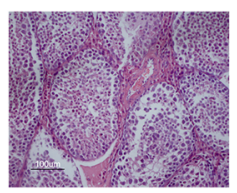

7 days

Fig. 3 Mouse model of cryptorchidism. a The normal (red circle) and cryptorchid (black circle) mouse testes. Photograph of testes of cryptorchidism group (first row) and control group (second row). $\mathbf{b}$ The testis index of cryptorchidism group and control group. Testis index = (testis weight/whole body weight) ${ }^{*} 100 \%$. c The histological comparison of testicular tissues between cryptorchidism group and control group. Bar $=100 \mu \mathrm{m}$. Con: control; UT: untreated side; T: treated side. ${ }^{*} P<0.05,{ }^{* *} P<0.01$ and ${ }^{* * *} P<0.001$

In addition, HE staining demonstrated the morphological changes of cryptorchid testes (Fig. 3c). The treated side testes retained normal spermatogenesis and showed no significant difference compared to the controls at 3 days after operation. However, cryptorchid testes showed obvious morphological abnormalities of seminiferous tubules at 7 days. From 14 to 28 days, we found that germ cells were progressively reduced and there was vacuolization in the seminiferous tubules of cryptorchid testes. At 28 days, only a small portion of germ cells remained in the basal part of the seminiferous tubules.

\section{miR-34c up-regulated Nanos2 in mouse model of cryptorchidism}

Similar to the expression pattern in humans, we found that the miR-34c level was significantly down-regulated in the treated side testes ( 3 days: Con vs $T=1.01 \pm 0.154$ vs $0.837 \pm 0.214, P=0.139$; UT vs $T=1.36 \pm 0.643$ vs $0.837 \pm 0.214, P=0.089$. 7 days: Con vs $T=1.04 \pm 0.330$ vs $0.467 \pm 0.308, P=0.011$; UT vs $T=0.966 \pm 0.376$ vs $0.467 \pm 0.308, P=0.031$. 14 days: Con vs $T=1.44 \pm 0.534$ vs $0.577 \pm 0.519, P=0.024 ;$ UT vs $\mathrm{T}=1.01 \pm 0.148$ vs $0.577 \pm 0.519, P=0.101 .21$ days: Con vs $T=1.20 \pm 0.750$ vs $0.210 \pm 0.234, P=0.022$; UT vs $T=0.984 \pm 0.211$ vs
$0.210 \pm 0.234, P<0.001 .28$ days: Con vs $T=1.21 \pm 0.721$ vs $1.03 \pm 0.628, P=0.625$; UT vs $T=2.03 \pm 0.343$ vs 1.03 $\pm 0.628, P=0.007$ ) (Fig. 4a). The mRNA level of Nanos2 was significantly up-regulated in the treated side testes of the cryptorchidism group at 7 and 21 days (3 days: Con vs $T=1.10 \pm 0.476$ vs $1.30 \pm 0.981, P=0.671$; UT vs $T=0.538 \pm 0.291$ vs $1.30 \pm 0.981, P=0.122$. 7 days: $C o n$ vs $T=1.29 \pm 1.03$ vs $5.39 \pm 2.80, P=0.008$; $\mathrm{UT}$ vs $\mathrm{T}=1.29$ \pm 0.639 vs $5.39 \pm 2.80, P=0.007$. 14 days: Con vs $\mathrm{T}=1.04$ \pm 0.317 vs $1.47 \pm 0.714, P=0.221$; UT vs $T=1.00 \pm 0.658$ vs $1.47 \pm 0.714, P=0.264$. 21 days: Con vs $T=1.13 \pm 0.623$ vs $1.23 \pm 0.540, P=0.765$; UT vs $T=0.579 \pm 0.229$ vs 1.23 $\pm 0.540, P=0.022$. 28 days: Con vs $T=1.12 \pm 0.578$ vs $1.50 \pm 0.467, P=0.244$; UT vs $T=1.16 \pm 0.430$ vs $1.50 \pm$ $0.467, P=0.215$ ) (Fig. 4b). Furthermore, Western blotting also validated that the protein level of Nanos2 was significantly increased in the treated side testes of the cryptorchidism group (Fig. 4c).

\section{SSC homeostasis disrupted in mouse model of cryptorchidism}

SSCs are capable of self-renewal and differentiation because of tissue homeostasis [21]. To investigate whether the abnormal expression of the miR-34c/Nanos2 pathway 


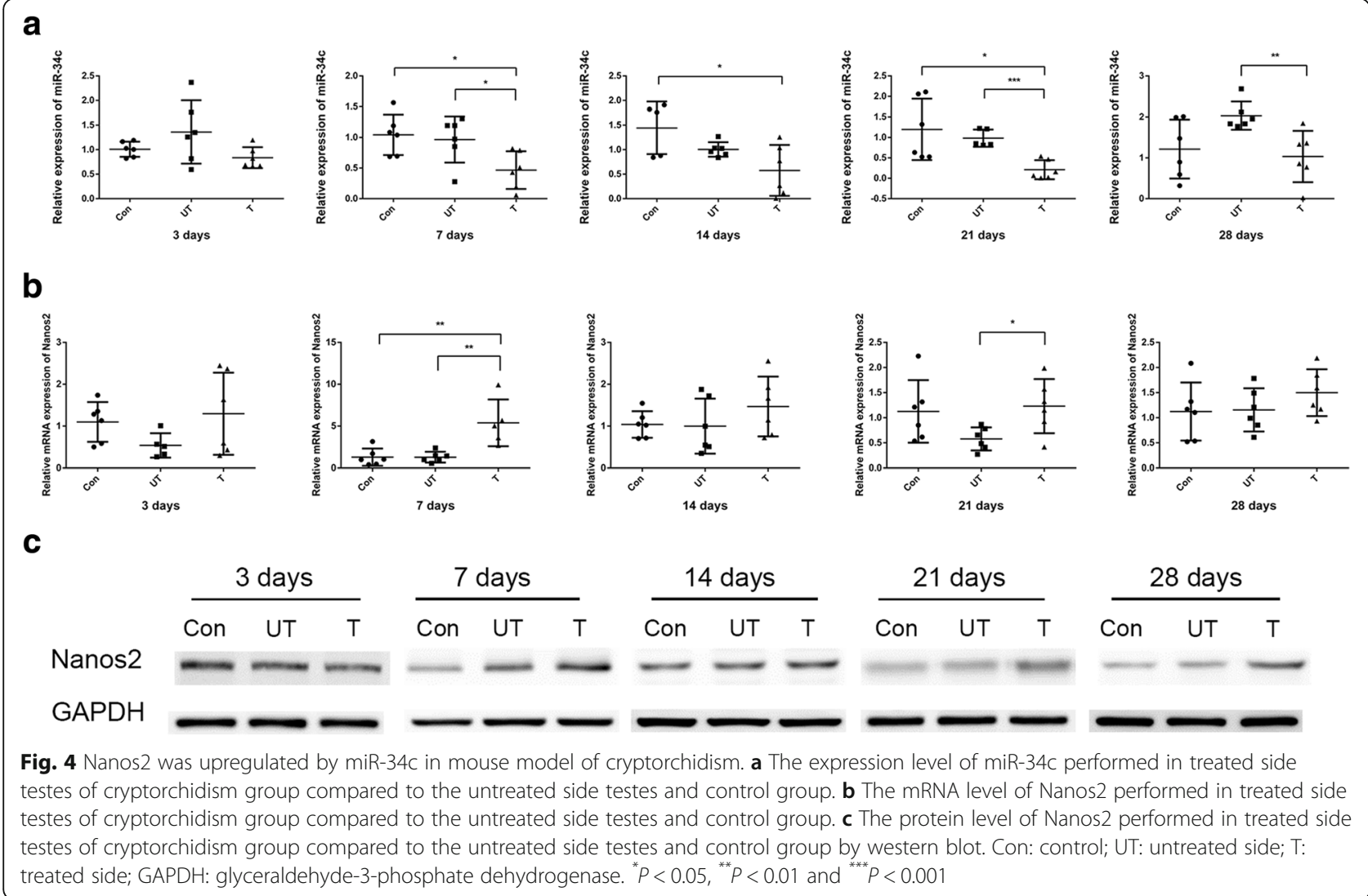

affects SSC homeostasis in cryptorchid testes, we analyzed the number of PLZF positive spermatogonia in the seminiferous tubules. By counting 80 seminiferous tubules from at least 20 independent microscopic fields, we found that the number of PLZF positive spermatogonia significantly increased in the treated side testes of the cryptorchidism group at 14 days after operation. Interestingly, the PLZF positive spermatogonia in the treated side testes of the cryptorchidism group showed no significant change as compared with the other two groups at 28 days after operation (14 days: $\left.P_{(\text {Con vs T) }}<0.001 ; P_{(\mathrm{UT} \text { vs T) }}<0.001\right)$ (Fig. 5a, b).

a

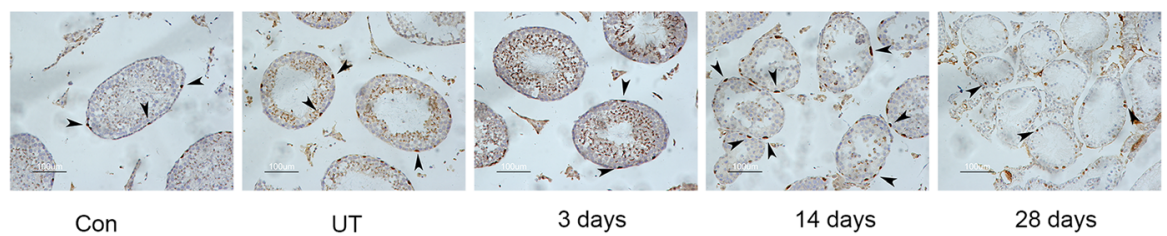

b

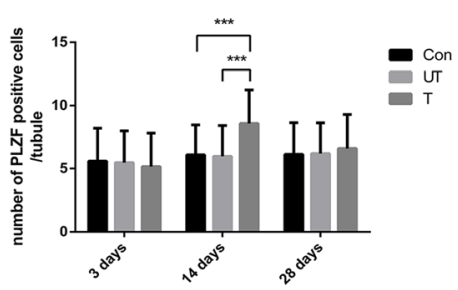

Fig. 5 SSC homeostasis was disrupted in mouse model of cryptorchidism. a Immunohistochemical staining of testicular tissue with antibody to PLZF. PLZF positive SSCs were indicated by black arrowhead. $\mathbf{b}$ The number of PLZF positive spermatogonia in treated side testes of cryptorchidism group compared to the untreated side testes and control group. Bar $=100 \mu \mathrm{m}$. Con: control; PLZF, promyelocytic leukemia zinc finger; SSCs, spermatogonial stem cells; UT: untreated side; T: treated side. ${ }^{* * *} P<0.001$ 


\section{Discussion}

Spermatogenesis is a complex, highly organized process in which post-transcriptional regulation is indispensable [22]. It is well known that miRNAs are one of the most common agents involved in the post-transcriptional regulation of genes. There are some other miRNAs, such as miR-210, that have been shown to be up-regulated in the testicular tissues of patients with cryptorchidism. miR-210 functions in spermatogenesis by targeting NR1D2 [19]. In the present study, we demonstrated that miR-34c was markedly reduced in the testicular tissues of patients with cryptorchidism. Subsequently, a surgery-induced mouse model of cryptorchidism was established to explore the roles of miR-34c in impaired spermatogenesis of cryptorchidism. Our surgery-induced mouse model of cryptorchidism reveals several key histological features, including morphological abnormalities of seminiferous tubules and an almost absence of mature spermatozoa, which is suitable for research on cryptorchidism. In accordance with the results in humans, miR-34c was significantly down-regulated in the mouse model of cryptorchidism. Recent studies have reported that miR-34c is involved in the proliferation, apoptosis, invasion, and prognosis of multiple malignancies. Here, we propose that miR-34c may serve as a novel target for treatment of infertility caused by cryptorchidism.

Nanos2 is a germ cell-specific gene that encodes an evolutionarily conserved RNA binding protein. In the embryonic period, Nanos 2 promotes the development of male primordial germ cells (PGCs) and inhibits them from meiosis $[23,24]$. In the process of spermatogenesis of testes, Nanos 2 suppresses the differentiation of SSCs and maintains the stability of the SSC pool [7]. Knockout of Nanos 2 causes the apoptosis of male PGCs, eventually leading to the loss of testicular germ cells and infertility of male mice [25]. Interestingly, the overexpression of Nanos 2 resulted in an increase in the number of PLZF positive spermatogonia by blocking the differentiation of SSCs rather than hyperproliferation or a decrease of apoptosis [8]. In the present study, we found that the mRNA level of Nanos 2 showed no statistical difference between the patients with cryptorchidism and the controls, maybe due to the lack of sufficient samples. In contrast to miR-34c, the protein level of Nanos 2 was significantly up-regulated in the cryptorchidism model. The mRNA level of Nanos 2 only increased at some time points in the model. We cannot rule out the possibility that the mRNA of Nanos 2 is stable with a long half-life time owing to its complex secondary structure. Generally, we found that miR-34c and Nanos 2 showed a negatively correlated expression pattern in the mouse model. However, we can't rule out the heat-sensitive expression of Nanos 2 itself. There is a possibility that Nanos 2 was sensitive to heat stress and directly up-regulated in cryptorchid testes, rather than involving miR-34c.

Stress granules (SGs) are conserved cytoplasmic particles that store non-translated messenger ribonucleoproteins (mRNPs) formed under stress conditions, such as heat stress, hypoxia or insufficient nutrition supply [26-28]. In a previous study, the formation of SGs were observed in spermatogonia in the testes of mice incubated at $42{ }^{\circ} \mathrm{C}$ for 20 mins [29]. Nanos 2 is one of the important components of SGs and possesses the role of promoting mRNP assembly [7, 30]. In the present study, the surgery-induced mouse model of cryptorchidism, with the abnormal expression of miR-34c/Nanos2, emerged as a disruptor of SSC homeostasis. We propose that on the one hand up-regulated Nanos 2 is involved in the formation of SGs to prevent the apoptosis of SSCs induced by heat stress. On the other hand, up-regulated Nanos2 disrupts the balance between SSC self-renewal and differentiation and eventually damages the spermatogenesis of cryptorchid testes.

\section{Conclusions}

In the present study, we demonstrated that miR-34c was significantly decreased in testicular tissues of patients with cryptorchidism. In contrast to miR-34c, the protein level of Nanos2 was significantly up-regulated in the mouse model of cryptorchidism. The abnormal expression of miR-34c/Nanos2 disrupted the balance between the self-renewal and differentiation of SSCs, eventually damaging the spermatogenesis of cryptorchid testes. Our research provides a new insight for the mechanism of male infertility caused by cryptorchidism. We propose that miR-34c may serve as a novel target for treatment of infertility caused by cryptorchidism.

\section{Abbreviations \\ Con: Control; Cry: Cryptorchidism; GAPDH: Glyceraldehyde-3-phosphate dehydrogenase; miRNAs: microRNAs; mRNPs: Messenger ribonucleoproteins; NC: Negative control; OA: Obstructive azoospermia; PGCs: Primordial germ cells; PLZF: Promyelocytic leukemia zinc finger; SGs: Stress granules; SSC: Spermatogonial stem cell; T: Treated side; UT: Untreated side}

\section{Acknowledgements}

We thank all subjects who provided the tissues for this study.

\section{Funding}

This study was funded by the National Natural Science Foundation of China (No. 81370749).

\section{Availability of data and materials \\ The datasets used and/or analyzed during the current study are available from the corresponding author on reasonable request.}

\section{Authors' contributions}

$\mathrm{ZH}$ and DT carried out the studies and drafted the manuscript. XD, PC, and DP participated in experiment work. JG, $Y Z$, and JM performed the statistical analysis. LZ and XZ conceived of the study and helped to draft the manuscript. All authors read and approved the final manuscript. 


\section{Ethics approval and consent to participate}

Written informed consent was obtained from all patients and this study was approved by the Ethics Review Board of The First Affiliated Hospital of Anhui Medical University (Approval No. Quick-PJ 2018-05-15). All animals were handled in accordance with the relevant guidelines, and all experimental procedures were approved by the Ethics Committee of Anhui Medical University (IRB approval number: LLSC20150346).

\section{Consent for publication}

Not applicable.

\section{Competing interests}

The authors declare that they have no competing interests.

\section{Publisher's Note}

Springer Nature remains neutral with regard to jurisdictional claims in published maps and institutional affiliations.

\section{Author details}

'Department of Urology, The First Affiliated Hospital of Anhui Medical University, Hefei 230022, China. ${ }^{2}$ Reproductive Medicine Center, Department of Obstetrics and Gynecology, The First Affiliated Hospital of Anhui Medical University, Hefei 230022, China. ${ }^{3}$ Anhui Province Key Laboratory of Reproductive Health and Genetics, Anhui Medical University, Hefei 230032, China. ${ }^{4}$ Anhui Provincial Engineering Technology Research Center for Biopreservation and Artificial Organs, Hefei 230022, China.

\section{Received: 27 June 2018 Accepted: 5 October 2018}

\section{Published online: 15 October 2018}

\section{References}

1. Sharlip ID, Jarow JP, Belker AM, Lipshultz LI, Sigman M, Thomas AJ, et al. Best practice policies for male infertility. Fertil Steril. 2002;77:873-82.

2. Agarwal A, Mulgund A, Hamada A, Chyatte MR. A unique view on male infertility around the globe. Reprod Biol Endocrinol. 2015;13:37.

3. Virtanen HE, Toppari J. Cryptorchidism and fertility. Endocrinol Metab Clin N Am. 2015:44:751-60.

4. Clavijo RI, Carrasquillo R, Ramasamy R. Varicoceles: prevalence and pathogenesis in adult men. Fertil Steril. 2017;108:364-9.

5. Kanatsu-Shinohara M, Shinohara T. Spermatogonial stem cell self-renewal and development. Annu Rev Cell Dev Biol. 2013;29:163-87.

6. Suzuki H, Sada A, Yoshida S, Saga Y. The heterogeneity of spermatogonia is revealed by their topology and expression of marker proteins including the germ cell-specific proteins Nanos2 and Nanos3. Dev Biol. 2009;336:222-31.

7. Zhou Z, Shirakawa T, Ohbo K, Sada A, Wu Q, Hasegawa K, et al. RNA binding protein Nanos2 organizes post-transcriptional buffering system to retain primitive state of mouse Spermatogonial stem cells. Dev Cell. 2015;34: 96-107

8. Sada A, Suzuki A, Suzuki H, Saga Y. The RNA-binding protein NANOS2 is required to maintain murine spermatogonial stem cells. Science. 2009;325:1394-8.

9. Bartel DP. MicroRNAs: genomics, biogenesis, mechanism, and function. Cell. 2004;116:281-97.

10. Hilz S, Modzelewski AJ, Cohen PE, Grimson A. The roles of microRNAs and siRNAs in mammalian spermatogenesis. Development. 2016:143:3061-73.

11. Tao WY, Wang CY, Sun YH, Su YH, Pang D, Zhang GQ. MicroRNA-34C suppresses breast Cancer migration and invasion by targeting GIT1. J Cancer. 2016;7:1653-62.

12. Li F, Chen $H$, Huang Y, Zhang Q, Xue J, Liu Z, et al. miR-34c plays a role of tumor suppressor in HEC1-B cells by targeting E2F3 protein. Oncol Rep. 2015;33:3069-74.

13. Wan Y, Shen A, Qi F, Chu J, Cai Q, Sferra TJ, et al. Pien Tze Huang inhibits the proliferation of colorectal cancer cells by increasing the expression of miR-34c-5p. Exp Ther Med. 2017;14:3901-7.

14. Liang X, Zhou D, Wei C, Luo H, Liu J, Fu R, et al. MicroRNA-34C enhances murine male germ cell apoptosis through targeting ATF1. PLoS One. 2012;7:e33861.

15. Bao J, Li D, Wang L, Wu J, Hu Y, Wang Z, et al. MicroRNA-449 and microRNA-34b/c function redundantly in murine testes by targeting E2F transcription factor-retinoblastoma protein (E2F-pRb) pathway. J Biol Chem. 2012;287:21686-98.
16. Comazzetto S, Di Giacomo M, Rasmussen KD, Much C, Azzi C, Perlas E, et al. Oligoasthenoteratozoospermia and infertility in mice deficient for miR-34b/c and miR-449 loci. PLoS Genet. 2014;10:e1004597.

17. Wu J, Bao J, Kim M, Yuan S, Tang C, Zheng H, et al. Two miRNA clusters, miR$34 \mathrm{~b} / \mathrm{c}$ and miR-449, are essential for normal brain development, motile ciliogenesis, and spermatogenesis. Proc Natl Acad Sci U S A. 2014;111:E2851-7.

18. Yu M, Mu H, Niu Z, Chu Z, Zhu H, Hua J. miR-34c enhances mouse spermatogonial stem cells differentiation by targeting Nanos2. J Cell Biochem. 2014:115:232-42

19. Duan Z, Huang H, Sun F. The functional and predictive roles of miR-210 in cryptorchidism. Sci Rep. 2016:6:32265.

20. Dou X, Gao J, Gao P, Tang D, Peng D, Mao J, et al. Association between RNAbinding protein Ptbp2 and germ cell injury in an experimentally-induced unilateral cryptorchidism murine model. PLoS One. 2017;12:e0186654.

21. Yu J, Lan $X$, Chen $X, Y u C, X u Y$, Liu $Y$, et al. Protein synthesis and degradation are essential to regulate germline stem cell homeostasis in Drosophila testes. Development. 2016;143:2930-45.

22. Kotaja N. MicroRNAs and spermatogenesis. Fertil Steril. 2014;101:1552-62.

23. Kato $Y$, Katsuki T, Kokubo H, Masuda A, Saga Y. Dazl is a target RNA suppressed by mammalian NANOS2 in sexually differentiating male germ cells. Nat Commun. 2016;7:11272

24. Suzuki A, Saga Y. Nanos 2 suppresses meiosis and promotes male germ cell differentiation. Genes Dev. 2008;22:430-5.

25. Tsuda M, Sasaoka Y, Kiso M, Abe K, Haraguchi S, Kobayashi S, et al. Conserved role of nanos proteins in germ cell development. Science. 2003; 301:1239-41.

26. Mitchell SF, Jain S, She M, Parker R. Global analysis of yeast mRNPs. Nat Struct Mol Biol. 2013;20:127-33.

27. Buchan JR, Kolaitis RM, Taylor JP, Parker R. Eukaryotic stress granules are cleared by autophagy and Cdc48/NCP function. Cell. 2013;153:1461-74.

28. Kedersha N, Ivanov P, Anderson P. Stress granules and cell signaling: more than just a passing phase? Trends Biochem Sci. 2013;38:494-506.

29. Kim B, Cooke HJ, Rhee K. DAZL is essential for stress granule formation implicated in germ cell survival upon heat stress. Development. 2012; 139:568-78

30. Zhou Z, Kawabe H, Suzuki A, Shinmyozu K, Saga Y. NEDD4 controls spermatogonial stem cell homeostasis and stress response by regulating messenger ribonucleoprotein complexes. Nat Commun. 2017;8:15662.

\section{Ready to submit your research? Choose BMC and benefit from:}

- fast, convenient online submission

- thorough peer review by experienced researchers in your field

- rapid publication on acceptance

- support for research data, including large and complex data types

- gold Open Access which fosters wider collaboration and increased citations

- maximum visibility for your research: over $100 \mathrm{M}$ website views per year

At BMC, research is always in progress.

Learn more biomedcentral.com/submissions 